\title{
IV. Karikaturen: Interdependenz von Bild, Schrift und Erzählung
}

\section{Universelle, länder- und sprachspezifische antinapoleonische Karikaturen}

Die Karikaturen stellen ein weiteres Medium dar, das in den Wirren der napoleonischen Eroberungspolitik weite Verbreitung fand, insbesondere in der letzten Phase der napoleonischen Ära $^{1}$. Dieses einst von der Kunstgeschichte wenig beachtete und von der Geschichtswissenschaft meist nur als Illustration verwendete Bildgenre erlangte in den letzten Jahrzehnten mehr Aufmerksamkeit. Wegen der politischen Brisanz der Karikaturen erscheint es besonders interessant, diese in die bereits dargestellten kommunikativen Strategien der Westphalen einzubetten ${ }^{2}$. Insbesondere die antinapoleonische Karikatur, die sich direkt auf Napoleons politische Handlungen, aber auch auf seine Privatsphäre bezog, wies ein breites Repertoire auf $^{3}$. Das Bildmedium hatte den Vorteil, ein europaweites Publikum anzusprechen, denn es konnte sich über Länder- und Sprachgrenzen hinwegsetzen $^{4}$. So fällt beispielsweise bei der bekannten Karikatur von James Gillray über die frischgebackenen Staatsoberhäupter Europas eine gleichwertige Behandlung des Königreichs Westphalen mit den anderen Staaten auf, die von Napoleoniden, Familienmitgliedern Napoleons, regiert wurden ${ }^{5}$. In dieser Karikatur aus dem Jahre 1806 tritt Napoleon als Bäcker auf, der frischgebackene Figuren der Napoleoniden aus dem Ofen holt ${ }^{6}$. Die Öffentlichkeit, die diese Karikatur erreichen wollte, beschränkte sich ganz offensicht-

1 Über die späte Produktionsphase der antinapoleonischen Karikaturen in Deutschland, die sich erst nach dem gelockerten Zugriff der napoleonischen Zensur im Jahre 1813 entwickelte, vgl. L'anti-Napoléon, S. 10, 14f.; LÜSEBRINK, REICHARDT, »Kauft schöne Bilder, Kupferstiche...«, S. 26.

2 Vgl. SieglersCHMIDT, Kommunikation und Inszenierung, S. 456; LÜSEBRINK, REICHARDT, »Kauft schöne Bilder, Kupferstiche...«, S. 25.

3 Vgl. SCHUlze, Die deutsche Napoleon-Karikatur; ClerC, La caricature contre Napoléon; EgGS (Hg.), Napoleon; SCHEFfler, UnVERfEHrT, So zerstieben geträumte Weltreiche; L'anti-Napoléon; MATHIS, BENOIT, Napoleon I.; CILLESSEN, REICHARDT u.a. (Hg.), Napoleons neue Kleider.

4 Vgl. L'anti-Napoléon, S. 63.

5 Die Radierung des englischen Karikaturisten Gillray ist betitelt »Tiddy-Doll the great French Gingerbread-Baker, drawing out a new Batch of Kings«. Vgl. SCHEFFLER, UNVERFEHRT, So zerstieben geträumte Weltreiche; L'anti-Napoléon, S. 8, 42 f.; OWZAR, Fremde Herrschaft, S. 76f.; vgl. ferner KeSSEMEIER (Hg.), Ereigniskarikaturen, S. 162.

6 Eine Karikatur, die ebenfalls die Schöpfung von napoleonischen Staaten zum Thema hat und die 1814 von einem anonymen westphälischen Zeitgenossen erwähnt wird, ist weit ordinärer. Sie stellte Napoleon auf dem Nachttopf dar, Könige produzierend, und war untertitelt: »Was hat wohl Bonapart gespeist, Dass so viel Könige er sch-?«. ANONYMUS, Die französische Garküche, S. 110. 
lich nicht allein auf deutsche Territorien. An eine ähnlich über Länder- und Sprachgrenzen hinweg zusammengesetzte Öffentlichkeit war wiederum gegen Ende der napoleonischen Ära, im Jahre 1814, die Karikatur "So zerstieben geträumte Weltreiche« adressiert, die den Seifenblasen pustenden Napoleon mit dem jungen König von Rom, seinem Sohn, zeigt. Auf den Seifenblasen sind die Namen der napoleonischen Satellitenstaaten zu lesen ${ }^{7}$.

Damit wird ein Sinnbild für die Vergänglichkeit der napoleonischen Staatsschöpfungen evoziert. Außerdem vermittelt die Karikatur wahrscheinlich eine moralisierende Botschaft so wie im Fall des »Homo Bulla« von Bartholomäus Bruyn dem Älteren ${ }^{8}$. Von der Karikatur der frischgebackenen Napoleoniden und der in Texten parallel aufkommenden Metapher des »neugebackenen Königreichs « 1806 bis zur Karikatur mit den Seifenblasen als Sinnbild der vergänglichen napoleonischen Staatsschöpfungen 1813 hielten sich zwei Konstanten: Zum einen konnte eine breite Öffentlichkeit europaweit über die gleichen Bilder angesprochen und zur Rezeption dieser Karikaturen über die napoleonische Herrschaft aufgefordert werden. Die Bilder waren zum anderen unmittelbar bei ihrer Visualisierung verständlich. Sicherlich war »das Bild [...], darüber besteht seit der Antike Konsens, durch eine Anschaulichkeit gekennzeichnet, die dem Begriff abgeht«, denn es hat die Fähigkeit, ein viel direkteres Verständnis als die Schrift zu erzeugen, die erst die Mühen des Lesens erfordert ${ }^{9}$. Allerdings sind Bilder nicht immer von direkt einleuchtender Aussagekraft: Manche fordern ihren Betrachtern mit einer rätselartigen Bild- und Textkombination zur eigensinnigen Deutung und zur sinnstiftenden Findigkeit auf ${ }^{10}$.

Die antinapoleonische Karikatur hatte jedoch nicht immer einen solch klaren universellen Charakter wie die beiden exemplarisch erwähnten Blätter ${ }^{11}$. Dem europaweiten Adressatenkreis mancher Karikaturen stehen an-

7 Vgl. LÜSEBRINK, REICHARDT, »Kauft schöne Bilder, Kupferstiche...«, S. 121; SCHEFFler, UNVERFEHRT, So zerstieben geträumte Weltreiche, S. 74f., 221-223.

8 Das ältere emblematische Motiv des seifenblasenden kleinen Jungen in der Darstellung von Bruyn wird aufgrund der darin enthaltenen moralisierenden Botschaft von Falkenburg als "visuelle Predigt« ausgelegt: FALKENBURG, Ikonologie und historische Anthropologie, S. 132. Der »Homo Bulla« von Bruyn wurde 2005 in der Ausstellung »Faszination Meisterwerk « des Germanischen Nationalmuseums in Nürnberg gezeigt, mit dem Hinweis auf die Seltenheit des Motivs und auf seine Bedeutung als Sinnbild des Sprichwortes von Desiderius Erasmus "Der Mensch ist eine Blase«. Leider ist dieses Exponat nicht in den Ausstellungskatalog aufgenommen worden. Vgl. Faszination Meisterwerk.

9 Tschopp, Rhetorik des Bildes, S. 96.

10 Vgl. L'anti-Napoléon, S. 6. Über eine ähnliche grundlegende Charakteristik der populären Emblemata-Bücher im 17. Jh. vgl. FALKENBURG, Ikonologie und historische Anthropologie, S. $131 \mathrm{f}$.

11 Über das Verhältnis von wenigen internationalen bzw. über die nationalen Grenzen in Europa hinweg argumentierenden Motiven gegenüber einer Vielzahl von stark national geprägten Motiven der Karikaturlandschaft vgl. L'anti-Napoléon, 
dere gegenüber, die sehr land- und/oder sprachspezifisch waren ${ }^{12}$. Eine Karikatur solcher Art, die im Jahre 1813 unter anderem im Königreich Westphalen kolportiert wurde, stellte folgende Szene nach:

L'empereur entre à Moscou à la tète d'une armée mourante de faim \& de froid - il s'est enveloppé d'une peau de bouc, \& porte sur la poitrine un ecrit, avec les mots,/ich habe diesen Bock selbst geschossen/J'ai tué ce bouc moi meme. Expression ou proverbe allemand ${ }^{13}$.

Napoleon über die deutsche Redensart und in deutscher Sprache eingestehen zu lassen, er habe mit seinem Russlandfeldzug einen Fehler begangen, war in mehrfacher Hinsicht ein Kunststück, das allein in einer Karikatur denkbar war.

Hier bediente die Karikatur ausschließlich ein deutschsprachiges respektive zweisprachiges Publikum, das mit der Redensart vertraut war ${ }^{14}$. Sie benutzte sogar die kodierte Botschaft einer deutschen Redensart, um Napoleon ins Lächerliche zu ziehen ${ }^{15}$. Wie Dagmar Freist für die Flugschriften, die in den Londoner Wirtshäusern des 17. Jahrhunderts im Umlauf kamen, bereits feststellen konnte, verrät der Rückgriff auf Sprichwörter aus der Alltagspraxis die Zugehörigkeit der Adressaten solcher Werke zur Volkskultur:

Vor allem bei einer weitgehend nichtlesefähigen Gesellschaft ist die Untersuchung von Öffentlichkeit an den Schnittstellen von Mündlichkeit und Schriftlichkeit von entscheidender Bedeutung. Die oft bildhafte Umschreibung von Sachverhalten un-

S. 9 f., 15, 63. Hier wird von einer besonders makabren Motivistik für den deutschen Sprachraum gesprochen. Vgl. ferner Cillessen, ReICHARDT u.a. (Hg.), Napoleons neue Kleider, S. $34 \mathrm{f}$.

12 Weitere Länderspezifitäten sind u.a. die Tradition, die Qualität und die Innovation der englischen Karikaturen. So zeichnen sich die Karikaturen aus England insbesondere durch ihre hohe Stichqualität und durch die ausgeprägte und fast avantgardistische Verwendung von Sprechblasen in den Karikaturen selbst aus.

13 RNB St. Petersburg, F 993 Arch. Westf., K. 11, Nr. 5737-5816, hier Nr. 5763: Schreiben von F. J.H. von Wolff, Generalpolizeikommissar der Hohen Polizei in Marburg, an J.F. M. de Bongars, Generalinspektor der Gendarmerie mit der Hohen Polizei beauftragt, Februar 1813. Vgl. RNB St. Petersburg, F 993 Arch. Westf., K. 19, Nr. 11 919-12 074, hier Nr. 12 011: Bericht von C[erfy], Polizeiagent in Kassel, an J. F. M. de Bongars, 18.7.1813. Die Redensart heißt so viel wie »einen Fehler machen «; »einen Bock schießen « bezieht sich auf einen alten Brauch der Schützengilden, wonach der schlechteste Schütze als Trostpreis einen Bock erhielt. Vgl. SCHEFFLER, UNVERFEHRT, So zerstieben geträumte Weltreiche, S. $100 \mathrm{f}$., $214 \mathrm{f} ., 251 \mathrm{f}$.

14 Das Interesse für französische Sprichwörter und ihre Entsprechungen in der deutschen Sprache scheint allerdings ausgeprägt gewesen zu sein, was eine Suche nach einer Übersetzung des deutschen Sprichwortes für die Französischsprachigen, die in Berührung mit der Karikatur kamen, möglich macht. Ein gesonderter Gattungszweig unter den Wörterbüchern konnte hier als Hilfsmittel dienen: Vgl. BELIN, Dictionnaire des Proverbes; SALZER, Recueil de proverbes.

15 Auch Königin Luise von Preußen widerfuhren französische Wortspiele auf ihre Kosten im Rahmen einer französischen antipreußischen Karikatur, vgl. König Lustik!?, Kat. 31. 
ter Einbeziehung von Sprichwörtern und die Anwendung von Schmähreden aus der Alltagspraxis auf Personen in Staat und Kirche lenken den Blick auf die deutlich von der Volkskultur geprägte Verbalisierung und Konzeptualisierung abstrakter Sachverhalte ${ }^{16}$.

Die Kombination von Bild und Text ist ein gängiges Mittel bei Karikaturen: Speziell im genannten Fall der Karikatur Napoleons wird der idiomatische Ausdruck des "geschossenen Bocks" versinnbildlicht; die angebotene symbolische Bildersprache wird jedoch durch das Einfügen einer Aufschrift um Napoleons Hals gestützt, damit die Interpretation der Karikatur durch seine Betrachter und Leser nicht fehlschlagen $\mathrm{kann}^{17}$. Die dadurch nachgewiesenen Bemühungen des Karikaturisten um Unmissverständlichkeit stehen zum Teil in Diskrepanz zur gleichzeitigen Nutzung einer kodierten Sprache, wegen der verlangten Vertrautheit mit der hier erforderlichen deutschen Sprache. Diese Strategie zielte darauf ab, sich einer Art Geheimsprache für einen subversiven Inhalt zu bedienen, die wahrscheinlich den Reiz der antinapoleonischen Kritik erhöhen sollte ${ }^{18}$. Die Karikatur setzte sich in diesem Fall auch über die Sprach- und Ländergrenzen hinweg, jedoch auf einer ganz anderen Ebene als im zuvor erwähnten Fall der frischgebackenen Napoleoniden: Dem französischsprachigen Napoleon wurden erstaunlicherweise deutsche Worte in den Mund gelegt. Während diese Karikatur mit den sprachlichen Differenzen spielt, versucht die erste, diese Differenzen durch die Verwendung des Bildmediums aufzuheben, um so ein breiteres Publikum zu erreichen. Beide Karikaturen gemeinsam betrachtet zeigen das große Potential des Mediums »Karikatur « für die Napoleongegner auf: Mal bedienten sie sich der latenten Universalität des Bildmediums, um unmissverständlich das größtmöglichste Publikum zu erreichen, mal ließen sie im Bild mithilfe des Textes, der Bildsprache und des dargestellten Sprachbildes eine kodierte Sprache einfließen, welche die mögliche Zahl der Adressaten der Karikatur einschränkte.

Die meisten Karikaturen waren zuerst antinapoleonisch, bevor sie antiwestphälisch waren. Einige Karikaturen waren jedoch sogar speziell für das westphälische Publikum gedacht oder für andere, für die der Modellstaatcharakter des westphälischen Staates ebenfalls ein wichtiges Thema war, so im Fall der Karikatur, in der eine deutsche Magd französische und westphälische Rechtsschriften wie »Stromb[ecks] Rechtswisch« dem Feuer übergibt.

16 FrEIST, Wirtshäuser, S. 212. Vgl. EISELEIN (Hg.), Die Sprichwörter.

17 Über die Interdependenzen von Sprachbild und Bildsprache im Fall von Flugschriften und Flugblättern vgl. TsCHOPP, Rhetorik des Bildes, S. $95 \mathrm{f}$.

18 Allerdings muss einschränkend bemerkt werden, dass der Autor der Karikatur für die wörtliche Übersetzung ins Französische und überhaupt für den Hinweis, dass er eine Redensart zitierte, selbst bewusst gesorgt hat. Dies beinhaltete einen Appell an das französischsprachige Publikum, sich über die Bedeutung der Redensart zu informieren. 
Johann Heinrich Ramberg wird als Autor dieser Karikatur vermutet ${ }^{19}$. Manche Karikaturen wandten sich an länder- und sprachspezifische Öffentlichkeiten, andere waren breiter angelegt; aus dem Kreis der ersteren waren einige in der Lage, eine breitere Öffentlichkeit anzusprechen. Eine eingehende Analyse einzelner Karikaturen, ihrer Entstehungsgeschichte und Interpretation durch die westphälische Bevölkerung würde an dieser Stelle zu weit führen. Vielmehr wird im Folgenden der Versuch unternommen, den lokalen Wirkungsradius von Karikaturen nachzuzeichnen und anhand der vorliegenden Polizeiakten die Berührung und den Umgang der westphälischen Bevölkerung mit antinapoleonischen Karikaturen zu reflektieren. Mehr als die Inhalte der Karikaturen interessiert hier der Umgang der Westphalen mit diesem Medium und dessen Stellenwert im weiten Spektrum der kommunikativen Möglichkeiten der westphälischen Bevölkerung.

\section{Karikaturen im Gespräch und in den westphälischen Polizeiberichten}

Eine in den untersuchten Polizeiberichten besonders häufig erwähnte Karikatur entstand während Napoleons Rückkehr vom Russlandfeldzug. Sie stellt den König von Rom, den Sohn Napoleons, dar, der diesem entgegenläuft und sagt, er habe während der Abwesenheit des Vaters laufen gelernt. Darauf erwidert Napoleon, er habe auch vor den Russen laufen gelernt ${ }^{20}$. In seinem Rapport vom 25. Februar 1813 berichtete der Polizeiagent Cerfy unter anderem über die Gespräche der Kasselaner: »Auch erzählen sich die Bürger, daß - als Napoleon nach Paris kam, lief ihm der König von Rôm entgegen, und sagte ich habe laufen gelernt, darauf soll Napoléon gesagt ha-

19 OWZAR, Fremde Herrschaft, S.68f. Für eine Abbildung der Karikatur vgl. BоTт (Hg.), Freiheit - Gleichheit - Brüderlichkeit, S. 563. Mit »Strombecks Rechtswisch" könnte eines von folgenden Werken gemeint sein: vgl. STROMBECK, Prozeßordnung; DERS., Rechtswissenschaft des Gesetzbuches Napoleons; vgl. ferner die Karikatur »Wollt ihr alle mich verlassen? «: vgl. BoudON, Le roi Jérôme, S. 592; König Lustik!?; vgl. die Karikatur von G. Moutard Woodward, »König 〉Jerry` verabreicht seine Untertanen westphälisches Wildbret« aus dem Jahre 1807: RIES, Gesänge Zions, S. 135; zur Abwesenheit dieser Karikatur im norddeutschen Raum vgl. OWZAR, Vom Gottesgnadentum zum Verfassungspatriotismus, S. 142.

20 Vgl. RNB St. Petersburg, F 993 Arch. Westf., K. 11, Nr. 5737-5816, hier Nr. 5763. Eine weitere Variante bildet folgende Situation ab: »le rhoi de rome ténu par les Lisieres et l'empereur dans un traineau poursuivi par les Cosaques, et par dessous voilà Comment on apprend aux français à marcher «. RNB St. Petersburg, F 993 Arch. Westf., K. 16, Nr. 9854-9858, hier Nr. 9855: Précis individuel et impartial du Caractere, des sentiments des principales perssonnes de cette Ville, Bericht von A. H. Léonnard, französischer Sprachlehrer in Uelzen, Werradepartement, an J. F. M. de Bongars, [2.2. 1813]. 
ben und ich auch $\aleph^{21}$. Bemerkenswert ist, dass die Karikatur eher als Erzählung denn als Bild in Kassel verbreitet gewesen zu sein scheint ${ }^{22}$. Dies überrascht nicht weiter, berücksichtigt man, dass die Karikaturen wie die illustrierten Flugblätter "zu einer semi-oralen, stark von Mündlichkeit geprägten vormodernen Form der Kommunikation gehören. [...] Nicht von ungefähr nannte ein Publizist der Revolutionszeit die Karikaturen eine Art 'gesprochene Schrift ( (écriture parlée`) ${ }^{23}$ «. Die ausgeprägte Eignung der Karikaturen zur Nacherzählung bewirkte mitunter, dass die Grenze zwischen Wirklichkeit und Fiktion für die Zeitgenossen verwischte. Die Erzählung von der Karikatur rückte, mehr als die bildliche Darstellung es gekonnt hätte, in der Vorstellungskraft der Kasselaner die Szene von Napoleons Empfang durch den Sohn in Paris noch näher an die plausible Realität heran. Dadurch, dass die Vorlage der Karikatur nicht nötig war, um ihren kritischen Inhalt zu transportieren, kamen selbst Personen, die sich aus Angst vor Repressionen sonst nicht aktiv an der Verbreitung von Karikaturinhalten beteiligt hätten, doch in Berührung mit ihr. Damit erweiterte sich der Rezipientenkreis der Karikaturen in ihren erzählten Versionen. In seinem »unterthänigen Bericht « gab der Polizeiagent Gade die gleiche Erzählung als »Räthsel « weiter, das ebenfalls in Alfeld, nahe Hildesheim, kursierte:

Ein Kaufmann aus hiesiger Gegend (den ich jetzt nachforsche) hat, denn hiesigen Kaufleuten in Alfeld, folgendes Räthsel aufgegeben - Worin hat Napoleon jetzt mit seinem Sohne die größte Ahnlichkeit. - Die Antwort hat er am Ende gesagt, wäre die - Napoleon sein Sohn lernte jetzt laufen - und Napoleon auch (er hat damit sagen wolle, Napoleon liefe vor die Russe weg, $-^{24}$.

Mal wurde die Karikatur als Erzählung wiedergegeben, mal als Witz oder Rätsel. Damit erlangten die Karikaturen einen noch weiteren Wirkungsradius als ihre Auflagezahlen es vermuten lassen: Billiger als über die Nacherzählung war eine Karikatur wohl nicht zu verbreiten. Auch die unteren

21 RNB St. Petersburg, F 993 Arch. Westf., K. 16, Nr. 9760-9796, hier Nr. 9783, Bericht von C[erfy] an J. F. M. de Bongars, 25.2.1813.

22 Auch in Uelzen, wo viele Truppen stationiert waren, sollen die Karikaturen als Erzählungen kursiert haben, so der Sprachlehrer Léonnard in seinem Bericht an den Polizeichef Bongars: »On Dit qu'il parcours plusieurs Karricatures, dont l'une représente le rhoi de rome ténu par les Lisieres et l'empereur dans un traineau poursuivi par les Cosaques, et par dessous voilà Comment on apprend aux français à marcher. La seconde Le Diable aumilieu des Diables avec ses paroles: on peut ou être mieux, je ne les ais pas vues«. RNB St. Petersburg, F 993 Arch. Westf., K. 16, Nr.9854-9858, hier Nr. 9855.

23 LÜSEBRINK, REICHARDT, »Kauft schöne Bilder, Kupferstiche...«, S. 28. Über das narrative Potential der Karikaturen vgl. L'anti-Napoléon, S. 8.

24 RNB St. Petersburg, F 993 Arch. Westf., K. 16, Nr.9852-9853, hier 9853, Bericht von Gade, Polizeiagent in Alfeld, Ockerdepartement, an J. F. M. de Bongars, 21.2.1813. 
und weniger bemittelten Schichten konnten ihr politisches Bewusstsein an den serzählten K Karikaturen schärfen, ohne Kosten für deren Kauf zu haben. Karikaturen wurden so zu einem höchst erschwinglichen und zugänglichen Medium, denn ihre Verbreitung war nicht abhängig vom Preis des Karikaturbogens. Durch das Weiterzeigen oder Weitererzählen im Gasthaus oder im Handelskontor kam auf ein Exemplar eine Vielzahl von Rezipienten. Durch den Erzählmodus wussten zudem die Verbreiter oftmals selbst nicht mehr, dass ihre preisgegebenen Erzählungen, Anekdoten oder Rätsel ursprünglich Druckbilder gewesen waren.

\subsection{Affäre Blumenthal oder die ausgedehnte mediale Vernetzung der Karikaturen}

Der Barbier Johann Ferdinand Blumenthal rühmte sich im Gespräch mit dem Kasseler Gastwirt Arendt und dem Bürger Gethmann im Februar 1813 allerhand subversiver Kunstwerke. Von diesen beiden angezeigt, wurde er nach seiner Verhaftung in einem Polizeiverhör zur Rede gestellt. Es wurde ihm vorgehalten, er habe gegenüber Gethmann geäußert, er sei der Urheber einer Karikatur über den König von Preußen und zeichne außerdem für eine andere Karikatur verantwortlich. Diese stelle den König von Rom und Napoleon dar, letzterer in einer Tonne versteckt ${ }^{25}$. Blumenthal wehrte ab und versicherte, es handele sich bloß um eine Verleumdung seitens seiner Denunzianten. Neben diesen beiden Karikaturen wurde ihm von der Polizei vorgeworfen:

Haben Sie sich nicht ferner geäußert, daß Sie auch noch Verfasser von einem Stücke wären, so der Feldzug des Kaisser von Rußland zum Gegenstand gehabt, und worin das Gespräch zwischen dem Kaiser und dem König von Rom vorkömmt nämlich: Vater während Deiner Abwesenheit habe ich laufen gelernt. (L'Empereur) Ich auch habe von den Russen lauffen lernen? ${ }^{26}$

Worauf Blumenthal entgegnete, diese Geschichte sei ihm zuerst von Arendt erzählt worden. Er bestritt, sich für den Urheber derselben ausgegeben zu haben. Hier fällt auf, dass das Motiv der schon oben erwähnten Karikatur in den Augen Blumenthals und der Polizei als "Stück« galt. Blumenthal wurde außerdem beschuldigt, sich gerühmt zu haben, Verfasser einer Schmähschrift gewesen zu sein: "Haben sie sich nicht kurz vor ihrer Arrestation gerühmt, Verfasser eines Liebelle zu sein, welches bei Gelegenheit eines Nationalfestes auf Napoleonshöhe gegen den H. Maire v. Canstein erschie-

25 Vgl. RNB St. Petersburg, F 993 Arch. Westf., K. 17, Nr.10 736-10 759, hier Nr. 10 742: Verhörprotokoll von J. F. Blumenthal, 25. 2. 1813.

26 Ibid. 
nen? $«^{27}$ Auch in diesem Fall wehrte sich Blumenthal gegen die Anschuldigung und wusste die gardes du corps Weiland und Schencke als mutmaßliche Verfasser anzugeben ${ }^{28}$. Die Ermittlungen gegen den Barbier Blumenthal als mutmaßlichen Karikaturisten und Pamphletisten ergaben, dass er aus Königsberg stammte, Chirurg beim Blüchner Corps in Stolpe bei Danzig gewesen war und nach dessen Niederlage bei Lübeck in Gefangenschaft nach Nancy kam. Nach seiner Entlassung führte ihn sein Weg durch das Königreich Westphalen, wo er wahrscheinlich im Jahre 1807 entschied, sich in Kassel als Barbier niederzulassen. Neben dieser Tätigkeit vertrieb er Lotteriebillets ${ }^{29}$. Bald erkannte die Polizei, dass Blumenthal nicht $\mathrm{zu}$ den vielseitig tätigen Opponenten der napoleonischen Herrschaft gehörte, die sich mal als Karikaturist, mal als Pamphletschreiber ausgaben, sondern dass sie es mit einem Angeber zu tun hatte, bestenfalls mit einem, der die Rache seiner Denunzianten infolge eines Streites im Gasthaus zu spüren bekam. Aber selbst wenn Blumenthal nicht der Karikaturist und Pamphletist war, für den er sich gern ausgab oder als den man ihn gern von der Polizei verfolgen lassen wollte ${ }^{30}$, so zeigt seine Affäre, dass die westphälischen Bürger über das weite Register der Ausdrucksmittel gegen die napoleonische Herrschaft verfügten und durchaus bereit waren, sich dieser gelegentlich zu bedienen. Blumenthals Vergehen belief sich letzen Endes darauf, vor langer Zeit Geld gefälscht zu haben und zum Zeitpunkt seiner Verhaftung Besitzer eines Buches gewesen zu sein, das von Revolutionen und Aufruhr handelte und nach dem Vorfall konfisziert wurde ${ }^{31}$. Das Urteil des Generalinspektors der Gendarmerie, Bongars, fiel entsprechend aus:

27 Ibid. Vgl. Hassenpflug, Jugenderinnerungen, S. 144; KEIM, "Savoir vivre«, S. 138.

28 Es ist im Übrigen interessant, dass ausgerechnet während eines westphälischen Nationalfestes auf Napoleonshöhe eine solche Schmähschrift im Umlauf kam: Eine offiziell einberaumte Öffentlichkeit wurde verwendet, um antiwestphälische Inhalte zu verbreiten und eine Gegenöffentlichkeit zu entfalten. Über den Festkult in westphälischer Zeit und seine Verknüpfung mit Traditionen aus den vormaligen Herrschaften des Königreichs Westphalen vgl. KNAUER, »Der Zukunft Bild sei die Vergangenheit«, u.a. S. 532; DERS., Monarchischer Festkult.

29 Vgl. RNB St. Petersburg, F 993 Arch. Westf., K. 17, Nr.10 736-10 759, hier Nr. 10 738: Schreiben Nr. 1402 von P. Mercier, Polizeipräfekt in Kassel, an J. F. M. de Bongars, 22. 2. 1813; vgl. ibid., Nr. 10 741: Schreiben von H. Lang, Polizeikommissar in Kassel, an Bock, Bürochef der Polizeipräfektur in Kassel, 22.2. 1813; ibid., Nr. 10 742: Verhörprotokoll von J. F. Blumenthal, 25.2. 1813.

30 Bemerkenswert ist hier ein ähnlich ausgeprägter Hang zur Verstellung, wie bei westphälischen Zeitgenossen wie Dreyssig oder Harckwitz festgestellt, um nur zwei zu nennen, die im Kapitel B II. (Spracherwerb) erwähnt wurden.

31 Die Bittschrift seiner Frau in französischer Sprache an Bongars spricht dafür, dass Blumenthal Opfer eines Streites im Gasthaus geworden war. Vgl. RNB St. Petersburg, F 993 Arch. Westf., K. 17, Nr. $10736-10$ 759, hier Nr. 10740 : Bittschrift der Ehefrau Blumenthal an J. F. M. de Bongars, o. Datum. 
on n'a rien trouvé chez lui [...] il ne resulte pas de son interrogatoire qu'il soit l'auteur des libelles à lui attribués; Celui à l'occasion d'une fête publique donné à Napoleonshöhe n'a pu être fait que par un homme instruit, qui possede des connaissances classiques, et quant à celui où l'on fait parler le Roi de Rome à son auguste père, j'ai tout lieu de croire que Blumenthal n'en est pas l'auteur ${ }^{32}$.

Blumenthal schien der Polizei nicht gebildet genug, um als Autor für die zitierten Pamphlete und Karikaturen gelten zu können. Bongars' Stellungnahme zeigt auch, dass selbst die Polizeiführung über die Karikatur, die den König von Rom und Napoleon gegenüberstellte, als Pamphlet sprach. In der Tat waren Karikaturen im deutschsprachigen Raum oftmals von Pamphleten begleitet: „Comme en Angleterre, la caricature était doublée par le pamphlet, et c'est l'Allemagne qui se montra la plus haineuse et la plus virulente $\aleph^{33}$. Diese Verwechslung Bongars', der von der Karikatur als »libellé« sprach, beweist, wie die Karikaturen, die oftmals Szenen oder Situationen nachstellten und durch diesen Inhalt leicht erläutert werden konnten, weit über die Existenz eines Druckbilds hinaus in Umlauf kommen konnten ${ }^{34}$. Durch die Verselbständigung der Nacherzählungen von den Vorlagen, die die Karikaturen darstellten, gewannen diese nicht zuletzt in den Augen ihrer Rezipienten an authentischem Potential; ihr vermeintlicher Wahrheitsgehalt steigerte sich graduell in dem Maße, wie sie sich nicht mehr unmittelbar auf das enge Medium der Karikatur zurückführen ließen ${ }^{35}$. Die Nacherzählungen ermöglichten eher, auf wahre Begebenheiten zurückzublicken, als wenn der kritische Inhalt eindeutig seinen Ursprung in einer Karikatur gehabt hätte. Die erzählten Karikaturen erlangten durch die Verselbständigung der kritischen Inhalte von ihrem Träger wahrscheinlich einem ausgesprochen breiten Wirkungsradius.

\subsection{Affäre Mathusius oder die Steigerung der kritischen Inhalte aus den Karikaturen}

Ein weiterer Grenzfall zur Interdependenz von Karikatur und Erzählung verdient erwähnt zu werden. Im April 1811 meldete Bongars dem Justizminister Siméon das Vorgehen gegen die Beteiligten eines staatsfeindlichen Kneipengesprächs:

je reconnois depuis longtems l'inutilité des Punitions contre les Bavardages et Déclamations des gens du peuple.

32 RNB St. Petersburg, F 993 Arch. Westf., Nr. 13 850, Registre Nr. 1 de correspondance du Bureau de la police secrète, 2. 1.-18. 4. 1813: Schreiben Nr. 496 von J. F. M. de Bongars an P. Mercier, 28. 2. 1813.

33 L'anti-Napoléon, S. 15, vgl. S. 7 f., 26 f. So ging z. B. die Karikatur »La poursuite des lapins« von Voltz auf ein Pamphlet zurück. Vgl. ibid., S. $26 \mathrm{f}$.

34 Das »libelle« war ein kurzes Schriftstück mit satirischem Charakter. Vgl. JOUHAUD, »libelle«.

35 Vgl. LÜSEBRINK, REICHARDT, »Kauft schöne Bilder, Kupferstiche...«, S. 24. 
Cependant il m'est qui deviennent plus importans, par l'objet contre lequel on déclame, et la sorte d'injure proferée-. En conséquence je crois devoir informer V. Ex. qu'il résulte d'une déclaration faite ce matin par un garde de police de retour d'un voyage à Göttingen que le $\mathrm{N}^{\mathrm{e}}$ Daniel Mathusius, garçon Perruquier a dit mercredi passé 17 de ce mois, dans l'auberge appellée Deutschauss à Göttingue devant plusieurs particuliers: [...] >Notre Roi Jerôme n'est pas un Roi, c'Est un Roi de fabrique et que voulez vous avec tel Roi, il n'est pas Roi né; Et l'Empereur des français est aussi un Empereur de fabrique, il faut enfin qu'ils abandonnent leur Pays $\mathbf{s}^{36}$.

Der Entstehungskontext dieses Vorwurfs lässt sich nicht mehr rekonstruieren, allerdings findet sich das Bild der Napoleoniden als "rois de fabrique« ebenfalls in der einleitend erwähnten und europaweit öffentlich bekannten Karikatur über die frischgebackenen Staatsoberhäupter Europas ${ }^{37}$. Ob dem Perückenmacherlehrling Mathusius die Karikatur bekannt war, lässt sich nicht mehr feststellen, jedenfalls besteht eine offensichtliche Korrelation zwischen Bildmedium und Erzählung, welche die enge Verzahnung beider Medien nahelegt. Die graphischen Illustrationen und die metaphorischen Aussagemuster bauten zum Teil auf exakt den gleichen Argumentationsstrategien auf ${ }^{38}$.

Im berichteten Wirtshausgespräch ging die Erzählung sogar über die Karikatur hinaus, denn selbst Napoleon wurde als ein »roi de fabrique« in Frage gestellt, was bei der Karikatur, in der er den Bäcker abgibt, nicht der Fall ist. Der kritische Inhalt einer Karikatur konnte in ihren Nacherzählungen neue Dimensionen annehmen: Das Medium "Karikatur « besaß die Fähigkeit, leicht Abstand zu seiner Materialität zu gewinnen und in andere Medien überzugehen, um tradiert zu werden. Der hier erkennbare Transfer von der Karikatur zur Erzählung, also vom Bildmedium zur Mündlichkeit, zeugt von der Durchlässigkeit des Mediums »Karikatur«. Das Bild trat zurück, sein Inhalt und seine Aussage wanderten in der Form des Geredes weiter und festigten sich womöglich zu einem »inneren Bild« für die westphälische Öffentlichkeit ${ }^{39}$. Als kollektiv gespeichertes Motiv und Gedankengut

36 GStA PK, V. HA, Nr. 690, Korrespondenzregister der Verwaltung der Hohen Polizei, 10. 11. 1810-9.5. 1811: Schreiben Nr. 5055 von J. F. M. de Bongars an J. J. Siméon, Justizminister, 19.4. 1811.

37 Vgl. SCHEFFLER, UNVERFEHRT, So zerstieben geträumte Weltreiche; OwZAR, Fremde Herrschaft, S. 76.

38 Vgl. Tschopp, Rhetorik des Bildes, S. 96.

39 Die Vertreter des »iconic turn« würden in diesem Fall von der Verfestigung zu einem »inneren Bild « sprechen. Vgl. u.a. KITTSTEINER, »Iconic turn«. Der "performative turn « könnte im Fall des Mediums "Karikatur « ebenfalls Anregungen bieten. Die Karikaturen, die oftmals konkrete Szenen oder Situationen inszenierten, besitzen durchaus einen performativen Charakter. Vgl. MARTSCHUKAT, PATZOLD (Hg.), Geschichtswissenschaft und »Performative Turn«. Über die Nähe mancher Karikaturen mit Sprechblasen zu den Pamphleten mit Dialogen und zum Singspiel vgl. folgende Aussage: »La caricature devient alors véritablement narrative, et se rapproche des pamphlets ou vaudevilles dont elle reprend 
bot es sich den Westphalen an, die in verschiedenen Situationen des Alltagslebens darauf zurückgreifen konnten, um weiterführende Sinndeutungen daran anzuschließen ${ }^{40}$.

Weitere Karikaturen avancierten sicherlich im Laufe der napoleonischen Ära zu kollektiv gespeicherten »inneren Bildern«. Die Karikatur »Triumph des Jahres 1813. Den Deutschen zum Neuenjahr 1814«, die auf dem Sprachbild des menschenfressenden Napoleons als Reaktion auf die katastrophale Entwicklung des Russlandfeldzugs für die Grande Armée basierte, stieg sicherlich zu einem solchen kollektiven »inneren Bild « auf ${ }^{41}$, zumal die Versinnbildlichung von Napoleon, dessen Expansionspolitik Soldatenleben kostete, nicht nur imaginiert werden konnte, sondern schmerzvoll in die eigene Erfahrungswelt zahlreicher westphälischer Familien einrückte, deren Angehörige nicht mehr zurückkehrten ${ }^{42}$.

\section{Verknüpfte Bildlichkeit, Schriftlichkeit und Mündlichkeit}

Ein weiterer Agentenbericht zeigt ebenfalls, dass das Zusammenspiel von Bild und Schrift bei Karikaturen einen entscheidenden Faktor darstellte und dass für die Polizei nicht immer ersichtlich war, welche Darstellungsweise die ursprüngliche gewesen war. Der Polizeiagent van Baerll berichtete:

Koszebue doit avoir fait de vers qui ne sont pas a l'avantage de l'E.[mpereur] des français, je crois qu'il en existe des exemplaires en ville, [...] le these en est que le.[mpereur] dormant d'un sommeil inquiet a mayence dans son lit de faste couronné de trophées et entourré de la renommée et de la victoire - enfin une estafete

les dialogues. C'est ici qu'intervient pleinement la mise en image. La caricature est alors une sorte de synthèse théâtrale, où l'image représente la scène et les acteurs«. Der Leser und Beobachter der Karikatur wird zum Zuschauer. L'antiNapoléon, S. 8.

40 Im Kapitel B VI. (Wappen) werden u.a. gebackene westphälische Wappen thematisiert: Die Querverbindung, die sich zwischen beiden Kapiteln in diesem Punkt am Rand der Untersuchung herstellen lässt, könnte auch zum Hintergrund der Westphalen gehört haben. Könnte das »innere Bild«, die Metapher und Karikatur des frischgebackenen Königreichs, nicht die Westphalen dazu verleitet haben, zeitkritisch die westphälischen Wappen nachzubacken? Auch wenn diese Backwaren nicht als Kritik gemeint waren, dürfte anzunehmen sein, dass manche Westphalen ihre Visualisierung mit dem geläufigen »inneren Bild « ihres frischgebackenen Königreichs konnotierten.

41 Vgl. OWZAR, Eine Nation auf Widerruf, S. 66; vgl. ferner L'anti-Napoléon, S. 6, 9, 15, $62 \mathrm{f}$.

42 Vgl. Buschmann, Carl (Hg.), Die Erfahrung des Krieges. Die Kategorie »inneres Bild« greift m.E. zu kurz angesichts der weitverbreiteten Trauer in den westphälischen Familien. Von Trauer und Betroffenheit zeugen folgende zeitgenössische Schriften: NiemeYer, Trost und Erhebung; Namentliches Verzeichniß; vgl. ferner [MIERZINSKY], Erinnerungen aus Hannover, 1843, S. 121 f.; MÜLLER, Kassel seit siebzig Jahren, Bd. 1, S. 54. 
arrive il se leve en sursaut; part, veu, emmener la victoire mais elle s'echappe le riedeau tombe alors et le buste de l'empereur de Russie prend la place - ce these fait soupçonner encore une estampe mais elle peut exister en dos de l'arme coaliser mais je m'e crois pas qu'elle a parue en Westphalie ${ }^{43}$.

Die Vermutung van Baerlls, wenn sie zutraf, dass die Verse August von Kotzebues einem Kupferstich, einem Holzschnitt oder einer Radierung entsprachen, sind für den Transfer von der Ikonographie zur Schriftlichkeit oder umgekehrt bezeichnend. Die Art, wie er darüber berichtete, zeigt, dass er die Karikaturen selbst nicht gesehen hatte, jedoch über diverse Nacherzählungen in die Lage versetzt wurde, Verse und Druckbild wiederzugeben mit allen dazugehörigen Räumlichkeiten und den darin stattfindenden Handlungen und Emotionen - für seinen Vorgesetzten inszenierte er die theatralische Szene erneut. Seine Mutmaßungen gingen weiter: Die Existenz eines entsprechenden Druckbildes lokalisierte er in den Territorien im Rücken der napoleonischen Armee, während er die Verbreitung der Verse im Königreich Westphalen bedauerte ${ }^{44}$. Die Verse oder ihre mündliche Nacherzählung waren leichter zu verbreiten als das Druckbild selbst. Dieses Quellenzitat macht damit auch eine Aussage über die Abhängigkeit, Interdependenz und Komplementarität der verschiedenen Medien ${ }^{45}$. Über das symbiotische Verhältnis zwischen sprachlich vermittelter und im Bild veranschaulichter Aussage im Fall der Flugschriften und Flugblätter im 17. Jahrhundert macht Silvia Serena Tschopp folgende Feststellung, die auch für die Karikaturen in der napoleonischen Ära gültig sein dürfte:

Piktoriale und sprachliche Aussage weisen [...] offenkundige Kongruenzen auf, bedingen sich gegenseitig: Ein adäquates Bildverständnis setzt in aller Regel die Kenntnis schriftlicher Überlieferung und des sprachlich vermittelten Kontexts voraus, auf

43 RNB St. Petersburg, F 993 Arch. Westf., K. 9, Nr. 4347-4351, hier Nr. 4351: Bericht von T. v[an] b[aerll], Polizeiagent, an J. F. M. de Bongars, 17. 9. 1813.

44 Über die Annahme, dass die Armeen der Koalition die Karikaturen zu Hunderten auf ihren Streifzügen in Deutschland verteilten, vgl. L'anti-Napoléon, S. 12.

45 Vgl. BurkhardT, WerkstetTer, Die Frühe Neuzeit als Medienzeitalter, S. 2; TsCHOpp, Rhetorik des Bildes, S. 95. Über die Multimedialität der illustrierten Flugblätter schreiben Lüsebrink und Reichardt: »Überhaupt sind die illustrierten Flugblätter keine fest abgrenzbare Gattung, sondern eine kombinierte Vermittlungsform im Wirkungszusammenhang der Printmedien vom Liedblatt und der Bekanntmachung in Plakatform über die Flugschrift bis hin zur Zeitung und zum Kalender; im Kommunikationsprozeß haben sie eine besonders enge komplementäre Funktion zu Flugschriften und Zeitungen. Während diese SchriftMedien meist mit einem leichten zeitlichen Vorsprung erschienen und eine breitere, oft aber auch diffusere, schnell veraltende Information liefern, erfüllt das Nachrichtenbild die ergänzenden Aufgaben sowohl der prägnanten Zusammenfassung und didaktischen Vereinfachung, der visuellen Vergegenwärtigung und des >Beweises` als auch der suggestiven Deutung und Wertung und kann sich so dem Gedächtnis nicht zuletzt des `Gemeinen Manns` tiefer und nachhaltiger einprägen als reine Texte«. LÜSEBRINK, REICHARDT, »Kauft schöne Bilder, Kupferstiche...«, S. 24. 
die eine bildliche Darstellung unmittelbar oder mittelbar rekurriert. Der Text wiederum bedient sich des Bildes, um den verbal abgesteckten Deutungsspielraum zu konturieren und um seiner Botschaft optische Prägnanz und damit stärkere Wirkung zu verleihen ${ }^{46}$.

Ein weiteres Indiz dafür, dass die Verbreitung von Karikaturen nicht ausschließlich an papierne Druckbilder gebunden war, ist neben den >nacherzählten Karikaturen` auch die zentrale Bedeutung von Gegenständen und Accessoires als Unterlage für Karikaturen.

\section{Karikaturen auf Alltagsgegenständen oder die zeitkritische materielle Kultur der Westphalen}

In den Polizeiakten wird von Karikaturen berichtet, die auf den verschiedensten Gegenständen abgebildet waren. Der Polizeiagent Cerfy informierte Bongars am 8. Februar 1813: "Zu Leipzig sol eine Caricatur, auf dosen gemahlt geben, wo Napoleon mit einem Stok im Schnee herum kratzt, und unten stehet, ich suche meine Armée. - Zu Bragwurden auch solche Caricaturverwerdiget ${ }^{47}$. Es scheint, dass eine Art Tarnung durch die Verwendung von Alltagsgegenständen zur Verbreitung von Karikaturen ihrem subversiven Inhalt besser gerecht wurde; zugleich wurden deren Besitzer in dieser Ansicht bestätigt, indem damit die Notwendigkeit einer Tarnung zur Zirkulation der staatskritischen Botschaft suggeriert wurde. »Auf die Napoleonischen Kriege nehmen verschiedentlich spöttisch-kritische Darstellungen auf den Dosendeckeln Bezug, die die Person Napoleons karikieren«, bestätigt Detlev Richter ${ }^{48}$.

46 TsCHOPP, Rhetorik des Bildes, S. $94 \mathrm{f}$.

47 RNB St. Petersburg, F 993 Arch. Westf., K. 16, Nr. 9760-9796, hier Nr. 9784: Bericht von C[erfy] an J. F. M. de Bongars, 8. 2.1813. Hier kann an die Affäre Grosse erinnert werden: Grosse hatte eventuell diese Karikatur oder für die Vertreter des »iconic turn « dieses »innere Bild « parat, als er am Rand des »Westphälischen Moniteur " eine Meldung über den Russlandfeldzug dahingehend kommentierte, dass die Soldaten wieder auftauchen würden, wenn der Schnee schmelzen würde. Zum »iconic turn«vgl. Anm. 39 und die Ausführungen über die Affäre Grosse, siehe Online-Kapitel über das Medium »Brief«, http://halshs.archivesouvertes.fr/PLCI-NAPOLEON (1. 1.2013).

48 RicHTER, Lackdosen, S.31. Die Lackdosen hielten, nachdem sie zuvor eine Exquisitware für den Adel und für höfische Kreise gewesen waren, ab Ende des 18. Jh. und bis Mitte des 19. Jh. nun auch in den wachsenden städtischbürgerlichen Kreisen Einzug und avancierten zu einem wesentlichen Statussymbol. Die Schnupftabakdose insbesondere wurde zum bekanntesten Markenartikel der Braunschweiger Lackmanufaktur Johann Heinrich Stobwasser. Über die Manufaktur Stobwasser vgl. RicHTER, Lackdosen; DERS., Stobwasser. Das Motivspektrum der Lackdosen war breit: Die Miniaturmalereien auf kleinstem Format machten keinen Halt z. B. vor der Darstellung gewimmel- und handlungsreicher Schlachtenszenen. Die Motive reichen von Alltagsszenen, Genremalereien und Bildnissen über Landschaften und Historie, Lyrik, Theater, An- 


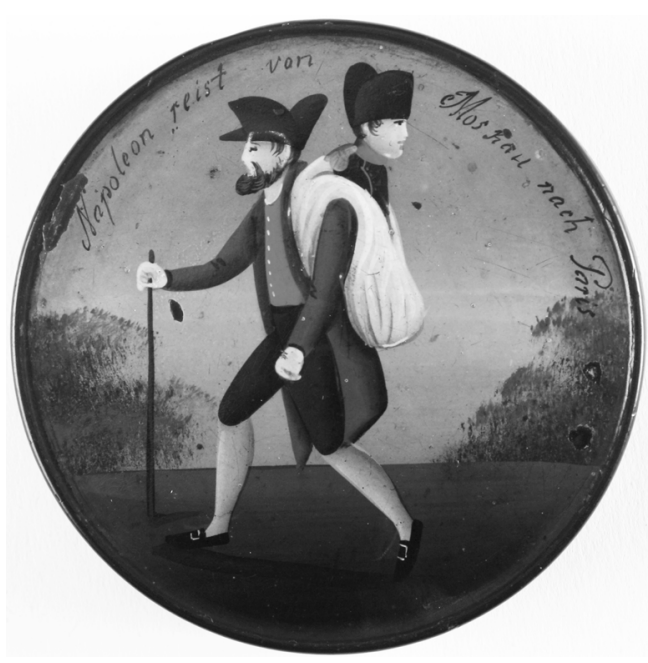

Abb. 17: Schnupftabakdose "Napoleon reist von Moskau nach Paris«, Manufaktur unbekannt, um 1815, $1,7 \times 9 \mathrm{~cm}$, Privatsammlung. Ein wandernder Russe mit Wanderstab trägt nach Napoleons Niederlage den französischen Kaiser im Rucksack zurück nach Paris.

Die Verbreitung von Karikaturen erfolgte durch eine Vielzahl von Möglichkeiten. Tabaksdosen oder Pfeifen mit ihren unverkennbar gesellschaftlichen und repräsentativen Funktionen waren als Träger für antinapoleonische Porträts, aber auch für Karikaturen besonders beliebt ${ }^{49}$. Die vor-

tike, Mythologie; sogar lüsterne Motive im Doppelboden prägen den Charakter etlicher Lackdosen. So gibt es auch Dosen, die Historie und Frivoles verbinden, wie etwa die Dose, die Napoleon mit seiner Mätresse darstellt. Vgl. RICHTER, Lackdosen, Kat. 288; vgl. ferner GLANZ, Historisches und Frivoles. Die Dosen mit erotischen Motiven sind vornehmlich bei Stobwassers Konkurrenz entstanden, allerdings sind auch solche Dosen in der Fabrik der pietistischen Familie Stobwasser entstanden. "Offenbar erfreuten sich Dosen mit pikantem Innenleben besonderer Nachfrage, insbesondere beim Militär, und versprachen daher guten Absatz. Die guten Verkaufsaussichten mögen die sittlichen Bedenken entkräftet haben«. RiCHTER, Stobwasser, S. 83.

49 Der anonyme Autor der »Garküche an der Fulda«, liefert einige Informationen über solche Dosen, die mit Porträts versehen waren, sowie ihre Verbreitungswege: »Oder hieß es nicht den Schatten an der Wand fürchten, als man die lackirten Dosen mit dem Bildnisse des Herzogs von Oels, Schills und Hofers, oder dickes dreifache Kleeblatt auf einer und derselben Dose confiscirte und einem Kaufmann auf der Casseler Messe mehrere Dutzend davon wegnahm, welche die Polizeiagenten doch nachher selbst wieder mala fide unter der Hand verkauften?« Ihm zufolge waren selbst die Polizeiagenten in den Kolportagehandel der subversiven Dosen nach deren Konfiszierung involviert. ANONYMUS, Die französische Garküche, S. 54. Über Karikaturen auf Dosen vgl. L'anti-Napoléon, S. 27, 32, 63, 70, 74. Die Mehrzahl der gesichteten Polizei- und Agentenberichte über die Pfeifen und Tabakdosen betreffen solche, die mit Porträts zum Andenken an antinapoleonische Helden verziert waren. Vgl. GStA PK, V. HA, Nr. 697, Briefecopiebücher der Verwaltung der Hohen Polizei zu Kassel, 1. 8.-26. 10.1811: Schreiben Nr. 3108 von J. F. M. de Bongars an F. T. de Guntz, Generalpolizeikommissar der Hohen Polizei in Braunschweig; ibid., Schreiben Nr.3123 von 
geschlagene Bezeichnung "Alltagsgegenstände« für diese außergewöhnlichen Träger der Karikaturen mit Geselligkeitscharakter sollte über deren Anziehungskraft als besondere Schmuckstücke nicht hinwegtäuschen sie hatten bereits als Sammler- und Liebhaberobjekte gedient. Ihre Qualifizierung als Gebrauchsgegenstände wäre korrekter ${ }^{50}$. Der Wunsch, etwas Außergewöhnliches zu besitzen ist für den Erfolg der Lackartikel ausschlaggebend $^{51}$.

Man reichte seine staatskritische Pfeife sicherlich mit verdecktem oder demonstrativem Stolz herum, zumal die französischen Einwanderer sich über die Rauchgewohnheiten der einheimischen Westphalen besonders erregen konnten ${ }^{52}$. Dadurch ergab sich eine Verdoppelung des staatskritischen Akts: die Karikatur, verbunden mit der missbilligten deutschen Rauchgewohnheit. Es dürfte anzunehmen sein, dass die darin enthaltene Provokation das soziale Ansehen der Besitzer in den Augen ihrer Gefährten erhöhte - zudem, wenn sie sich der Wachsamkeit der Polizeiüberwachung entzogen.

Der praktische Charakter lässt sich ebenfalls nicht leugnen: Eine Tabaksdose konnte man sicherlich leichter in die Hosentasche stecken und unauffällig herumreichen als ein empfindliches Druckbild - weniger kompromittierend war sie allerdings nicht. Bei seiner Tabaksdose konnte man jedoch auch wählen, wem man sie zeigte. Diese Gegenstände nahmen einen fes-

J.F. M. de Bongars an F.T. de Guntz; GStA PK, V. HA, Nr. 698, Briefecopiebücher der Verwaltung der Hohen Polizei zu Kassel, 28.10.1811-März 1812: Schreiben Nr. 3800 von J.F. M. de Bongars an F. J. H. von Wolff, 13. 12.1811; GStA PK, V. HA, Nr. 699, Briefecopiebücher der Verwaltung der Hohen Polizei zu Kassel, 4. 3.-7. 7. 1812: Schreiben Nr. 1152 von J. F. M. de Bongars an Mertens, Generalpolizeikommissar der Hohen Polizei in Göttingen, 18. 5. 1812; RNB St. Petersburg, F 993 Arch. Westf., K. 19, Nr. 11 919-12 074, hier Nr. 11 969: Rapport Nr. 86 von C. E[skuchen], Polizeiagent in Kassel, an J. F. M. de Bongars, 1. 6. 1813. Vgl. auch Thimme, Die inneren Zustände, Bd. 2, S. 185.

50 Klein führt zu Recht an: »Lackwaren waren Luxuswaren«, denn die Herstellung war recht aufwändig. KLEIN, Lackmanufaktur Stobwasser, S. 659, vgl. ferner S. 658 .

51 RiCHTER, Lackdosen, S. 16. Auch die französischen Einwanderer trugen zu diesem Markterfolg bei, denn auch sie waren von der Qualität der Stobwasserschen Lackartikel sehr angetan. Stobwasser, wie im Übrigen seine Konkurrenten, hielten in ihrem Motivrepertoire eine breite Auswahl an Porträtierten bereit, so dass man »jeder persönlichen Vorliebe gerecht wurde«. DERS., Stobwasser, S. 80, vgl. ferner S. 36f., $45 \mathrm{f}$.

52 Vgl. [MierZinSKY], Erinnerungen aus Hannover, 1843, S.41; [WOLFFradT], Denkwürdigkeiten, S. 381; KIRCHEISEN, König Lustig, S. 90; FABRE, Jérôme Bonaparte, S. 102. Tatsächlich scheinen die Streitigkeiten der Westphalen französischer und deutscher Herkunft über die Rauchgewohnheiten im öffentlichen und privaten Raum während der westphälischen Herrschaft als deutsch-französische kulturelle bzw. vermeintlich `nationalkulturelle` Unterschiede wahrgenommen und angesprochen worden zu sein. 


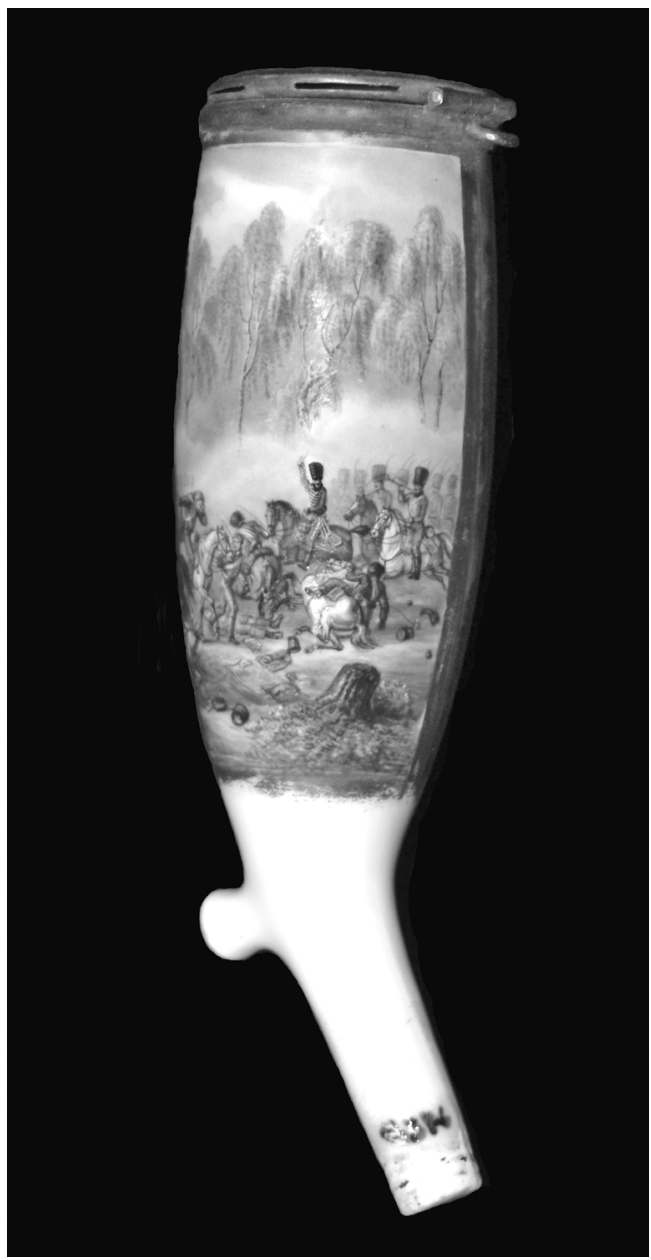

Abb. 18: Pfeifenkopf mit buntem Bild »Rückzug der Franzosen 1812« und Metalldeckel, nach 1812, Porzellan, $H: 12,5 \mathrm{~cm}, S M L$, Po 346.

ten Platz in den Attributen der Staatskritiker ein: Generalpolizeikommissar Wolff berichtet in seiner »Kurzen Darstellung«, wie Polizeiagenten mit ihnen ausgestattet wurden, um sich den »Räsoneurs«, die so unvernünftig waren, sich gegen die Staatsräson zu äußern, besser anzunähern und von ihnen als ihresgleichen angenommen zu werden ${ }^{53}$. Er zitiert den Bericht eines Kasseler Polizeiagenten aus dem Jahre 1812, den er zufällig vorgelegt be-

53 Über die Staatsräson vgl. BERDING, Napoleonische Herrschafts- und Gesellschaftspolitik, S.23, 79 f.; MAIER, Die ältere deutsche Staats- und Verwaltungslehre, S. 17; PUHLE, Das Herzogtum Braunschweig-Wolfenbüttel, S. 65; STOLLEIS (Hg.), Staat und Staatsräson; LÜDTKE, Einleitung: Herrschaft als soziale Praxis, S. $27 \mathrm{f}$. 
kommen haben will: »In Helm logiren zwei sehr verdächtige Fremde. Sie hüten sich gewaltig im Sprechen, werfen die Augen umher, und müssen also wohl beobachtet werden. Meine Schillsdose soll ihnen den Mund öffnen ${ }^{54}$. Erläuternd für den unerfahrenen Leser seiner legitimierenden »Darstellung « fügt Wolff zu den Schillsdosen hinzu: »Die Spione bekamen weggenommene Dosen mit dem Bildnisse Schills, Hofer, des Kaisers von Rußland, des Herzogs von Oels u., um damit den Fremden Tabak anzubieten, und sie zum Sprechen zu reizen $\aleph^{55}$. Demnach avancierten die besagten Dosen oder Pfeifen, ob sie mit einem Porträt eines Feldherrn, eines renommierten napoleonischen Gegners oder einer Karikatur versehen waren, zu Erkennungszeichen, die unmittelbar die politische Orientierung ihrer Träger verrieten.

Der folgende Bericht des Polizeiagenten Cerfy macht diese Funktion deutlich:

Den 28 v. Monats frühstükten der Obrist von Bauer und Lieut. von Hornstein im Adler zu Schmalkalten, ersteres rauchten eine Pfeifenkopf gannen mit dem Bildniß von Prinz Oehls - der andr mit ein Sinnbild wo ein Aug - und ein Ohr darauf stand, untstand - und schweigen, ich sagte zu dem Lieut. - was das bedeiten soll, darauf sagte er mir mann muß von den Grausamkeiten der grosen nichts sprechen, hernach sagte er wir gehen nach Haus, um uns wiederum zu organisiren, und den künftige Kinder werden mir der nehmliche Schiksal erleben ${ }^{56}$.

Bemerkenswert ist hier der Hinweis, dass die Dosen oder Pfeifen zunächst als politisches Erkennungszeichen für Gleichgesinnte unter Verschwiegenheit fungierten und ihre Träger es nicht mal mehr nötig hatten, »den Mund zu öffnen«, um in der Öffentlichkeit mit politischem Gerede aufzufallen. Ein anderer Anlass zum Herumreichen einer Tabaksdose wird vom westphälischen Sergenten Kroschke genannt, der im Auftrag seines Chefs verdeckt dem Jahrestag des Zusammenstoßes der westphälischen Truppen mit dem Herzog von Braunschweig-Oels am 1. August 1809 bei Ölper beiwohnte ${ }^{57}$ :

[Als wir] nach den Förster kammen, forderdten sie gleich ein Bodelligen Schnaps, und befahllen der Musicus an sie sollen spiellen es lebe Friedrich Wilhelm hoch, hoch Friedrich Wilhelm hoch. Der Musicus Backe welcher es auch spuelete, und die Geselschaft darzu gesungen hat, und jeder einen guden gb. bezahlt, und ich Ebenfals da ich mit darundter sass. Der Aufreger dafon war namens Schiedte, und Reimman

54 WolfF, Kurze Darstellung der Verwaltung der hohen Polizei, S. 18.

55 Ibid.

56 RNB St. Petersburg, F 993 Arch. Westf., K. 16, Nr. 9760-9796, hier Nr. 9784: Bericht von C[erfy] an J. F. M. de Bongars, 8. 2.1813.

57 Vgl. RNB St. Petersburg, F 993 Arch. Westf., K. 5, Nr. 2613-2655, hier Nr. 2617: Schreiben Nr. 687 von F. T. de Guntz an J. F. M. de Bongars, 5.8.1812; ibid., Nr. 2630: Schreiben Nr. 697 von F. T. de Guntz an J.F.M. de Bongars, 13.8. 1812. Über den Zusammenstoß zu Ölper vom 1. 8. 1809 vgl. HäNSELMANN (Hg.), Treue Bauern; DrEYER, Gefecht von Oelper; KorTZFLEISCH, Herzog Friedrich Wilhelm von Braunschweig; VoGES, Zur Geschichte des Gefechtes bei Oelper; MACK, Braunschweig; Rosendahl, Das Rätsel von Oelper; PuHLE, Das Herzogtum Braunschweig-Wolfenbüttel, S. 493, 502. 


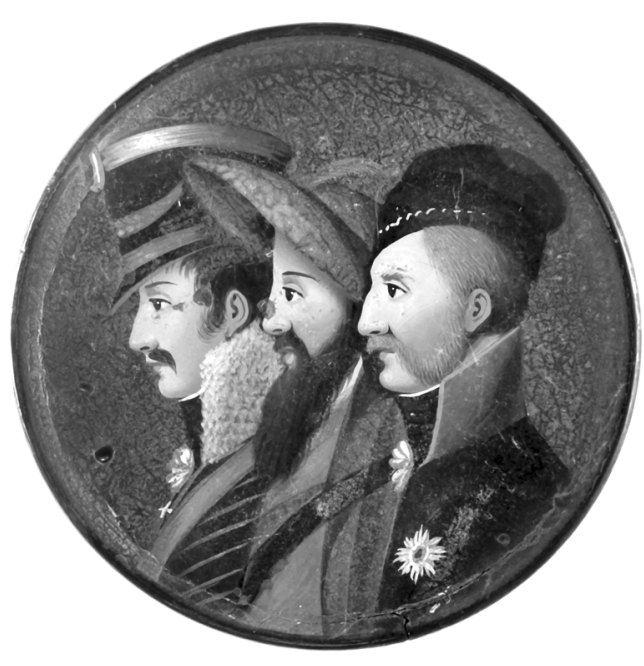

Abb. 19: Schnupftabakdose mit Profilbildnissen des Herzogs von BraunschweigOels, Andreas Hofers und Ferdinand von Schills, Manufaktur unbekannt, um 1815, $2,5 \times 9,5 \mathrm{~cm}$, Privatsammlung Braunschweig.

Die hier gutmütig und Seite an Seite dargestellten antinapoleonischen Volkshelden führten ihre bewaffneten Aktionen zwar aufeinander aufbauend, jedoch ohne weiterführende Verbindung miteinander durch. In der Nachwelt wurden die Verbindungslinien zwischen ihnen gern kultiviert ${ }^{58}$.

nimt das glaus und sagt jetz trunncke ich dem dem ich zu taencke des magens balde wieder einmal besuchen; aber nicht in so einer lage als die er uns hat verlassen missen sagt sömtliche geselschaft, es sind nun $<$ Jjafer $>$, der Schurdte kriegt die Schauhtaback Tohse raus und giebt sie herum wo sich der Herzog drauf befand, da sie nun rum war sagten sie dreistes auch Werth das wir in die Ehrre anthün, er soll auch 2 Teller liegen, es [werden] 2 Teller genommen und wurde auf jeden ein Theil der Tohsse gelegt was und gibt, jetz hiest <Sfast $>$ aus alle alte Deudsche sollen leben, und auser alter Primas hier, armlich des Herzog den sie for sich hadten ${ }^{59}$.

Bei diesem Jahrestag, reichlich mit »Schnaps« begossen, wie der Sergent Kroschke hervorgehoben in lateinischer Schrift berichtet, standen die Tabakdosen mit dem Porträt des Herzogs von Braunschweig-Oels im Mittelpunkt, auf zwei Tellern erhöht, damit des Herzogs auch gehörig gedacht und für ihn gesungen werden konnte.

Was die Kosten anbelangt, so bedeuteten die Karikaturen auf den Dosen sicherlich eine größere Investition als die Druckversion, auch wenn gravierende Unterschiede in der Qualität der Ausführung bestanden ${ }^{60}$. Edle Ausführungen von tabatières, beispielsweise mit den Porträts Napoleons und Jérômes und mit Diamanten besetzt, wurden im Zuge der Plünderungen

58 Eine weitere Schnupftabakdose mit den gleichen drei Porträts, jedoch mit kämpferischerer Ausrichtung, ist abgebildet in: König Lustik!?, Kat. 450.

59 RNB St. Petersburg, F 993 Arch. Westf., K. 5, Nr. 2613-2655, hier Nr. 2631: Rapport von Kroschke, Sergent, an F. T. de Guntz, 1. 8. 1812.

$60 \mathrm{Im}$ Zuge der napoleonischen Kriege war die Nachfrage nach Lackarbeiten mit Darstellungen aus den Befreiungskriegen, von Schlachtenszenen über Porträts Napoleons und der antinapoleonischen Feldherren so groß, dass die Manufaktur Stobwasser ihre Produktion auf schnellere Vervielfältigungstechniken umstellte. Vgl. Richter, Stobwasser, S. 78. 
während des Schlossbrands in Kassel gestohlen: Die Pfeifen und Dosen mit Porträts gehörten zu den Requisiten des königlichen Schatzes ebenso wie sich qualitativ variierende Ausführungen im Besitz der Westphalen fanden ${ }^{61}$.

Über die verschiedenen erhältlichen Karikaturen-Produkte, von der Nacherzählung über das klassische Druckbild bis hin zu den Darstellungen auf Alltags- oder Geselligkeitsgegenständen, die auf dem >Kommunikationsmarkt` der Westphalen angeboten wurden, öffnet sich ein kommunikatives Spektrum, das offensichtlich in verschiedenen Gesellschaftsschichten Geltungsanspruch erlangen konnte.

Eine edle Ausführung stellte wahrscheinlich als Unikat die Pfeife dar, die der Göttinger Student König für einen Freund extra anfertigen ließ und die einen lokalen Streit zwischen zwei Burschenschaften darstellte, um an diese verbotenen Institutionen zu erinnern ${ }^{62}$. Generalpolizeikommissar Mertens berichtete dazu nach Kassel:

Il m'étoit rapporté que le Sr. König, étudiant de cette université avoit fait faire une tête à pipe ornée d'une peinture laquelle represantoit un duel entre des étudiants et avec la devise svirtute duce comite fortuna rau dessous de laquelle beaucoup de noms étoient peints.

Je me fis exhiber cette tête de pipe et j'appercus bientôt que la peinture se rapporte à un duel qui a eu lieu le 19 Juillet 1812 entre la société des étudiants de l'ancien pays de Brême et celle des étudiants de l'ancien pays de Hesse et que les noms sont ceux des personnes qui à cette époque se trouvoient être membres de la société des étudiants de l'ancien pays de Brême.

Cette société étant supprimée comme toutes les autres, cette tête de pipe ne paroit plus avoir quelque valeur, mais conservant du moins la memoire du temps florissant de la société j'ai fait au possesseur la défense formelle d'en faire un usage quelconque. Il m'a cependant assuré qu'il ne l'avoit pas fait faire pour en faire usage mais pour en faire cadeau à un ami qui depuis cinq mois a quitté l'université ${ }^{63}$.

Die Szene war mit einem lateinischen Motto unterlegt, das den Polizeibeamten nicht unmittelbar einleuchtete. So schrieb der ebenfalls involvierte Polizeikommissar Haas dazu: »Le motto paroit montrer qu'il s'agit d'une

61 Vgl. GStA PK, V. HA, Nr. 698: Rundschreiben Nr. 3669 von J. F. M. de Bongars an die Generalpolizeikommissare der Hohen Polizei, 28.11.1811; HePpe, Das Schloß der Landgrafen von Hessen, S. 291. Für die Innenausstattung des Braunschweiger Schlosses wurden von König Jérôme Lackartikel der Manufaktur Stobwasser in Auftrag gegeben. Vgl. RICHTER, Stobwasser, S. $45 \mathrm{f}$.

62 Vgl. u.a. RNB St. Petersburg, F 993 Arch. Westf., K. 14, Nr. 8041-8158, hier Nr. 8098: Schreiben Nr.91 von Haas, Polizeikommissar in Münden, Fuldadepartement, an J. F. M. de Bongars, 28. 2. 1813; RNB St. Petersburg, F 993 Arch. Westf., Nr. 13 850: Schreiben Nr. 470 von J. F. M. de Bongars an Haas, 26. 2. 1813; ibid., Schreiben Nr. 475 von J. F. M. de Bongars an Mertens, 26. 2. 1813.

63 RNB St. Petersburg, F 993 Arch. Westf., K. 14, Nr. 8041-8158, hier Nr. 8099: Schreiben Nr. 163 II. Sekt. von Mertens an J. F. M. de Bongars, 24.2. 1813. Vgl. ferner Thimme, Die inneren Zustände, Bd. 2, S. 307-313. 
société politique « ${ }^{64}$. Bemerkenswert ist an diesem Fall auch, dass der bereits erwähnte Geselligkeitscharakter offensichtlich mit Schenkungspraktiken gepaart war ${ }^{65}$. Die Besitzer dieser Gegenstände hingen in vielen Fällen sehr an ihnen, wie die Braunschweiger Polizeibeamten bei deren Konfiszierung feststellen mussten: Ein Tabakdosenbesitzer wurde sogar handgreiflich, weil er seine Dose nicht hergeben wollte ${ }^{66}$. Bongars musste einen Generalpolizeikommissar daran erinnern, er solle die besagten Gegenstände trotz wiederholter Anfrage ihrer Besitzer nicht wieder zurückgeben, sondern diese allesamt vielmehr zu ihm nach Kassel übersenden. Von den Westphalen in breitem Umfang rezipiert ist auch der bereits erwähnte Fall eines Konskribierten, der angeblich von einem Gendarmen in Marburg erschlagen wurde, weil er sich weigerte, die Pfeife aus dem Mund zu nehmen ${ }^{67}$. Die Beziehung der Westphalen zu ihren Pfeifen war sicherlich emotional geladen: Man starb auch patriotisch durchaus mit der Pfeife in der Hand, so ein Aufständischer, der infolge des Dörnberg-Aufstands gegen Jérôme hingerichtet wurde: »ein alter Oberst [starb] mit der Tabakspfeife in der Hand unter dem Rufe: `Es lebe der Kurfürst!«« 68

Einige dieser Objekte wurden sicherlich im Königreich Westphalen produziert - Braunschweig wird als Produktionsort für Pfeifen mit antinapoleonischen Porträts in den Polizeiberichten genannt ${ }^{69}$. Andere wurden über Kolportagehandel ${ }^{70}$ aus dem Ausland importiert, so beispielsweise aus Sachsen ${ }^{71}$, wo auch der Student König den Auftrag zu seiner speziell angefertigten Pfeife aufgegeben hatte ${ }^{72}$.

64 RNB St. Petersburg, F 993 Arch. Westf., K. 14, Nr. 8041-8158, hier Nr. 8101: Schreiben Nr. 84 von Haas an J. F. M. de Bongars, 25. 2. 1813. Vgl. u.a. THIMME, Die inneren Zustände, Bd. 2, S.314f. Weiterführend vgl. SACHSE, Göttingen im 18. und 19. Jh.; FREVERT, Bürgerlichkeit und Ehre; FÜRBRINGER, Metamorphosen der Ehre; FREVERT, Ehrenmänner.

65 Über weitere Hinweise zu den Schnupftabakdosen, Dosen und Pfeifenköpfe als Geschenk und Freundschaftsband vgl. RiCHTER, Stobwasser, S. $45 \mathrm{f}$.

66 Vgl. GStA PK, V. HA, Nr. 697: Schreiben Nr. 3136 von J. F. M. de Bongars an F. T. de Guntz.

67 Vgl. Thimme, Die inneren Zustände, Bd. 2, S. 183.

68 PRÖHLE, Die Fremdherrschaft, S. 17.

69 Vgl. GStA PK, V. HA, Nr. 698: Schreiben Nr. 58 von J. F. M. de Bongars an F. T. de Guntz, 10.1.1812.

70 Vgl. ibid., Schreiben Nr. 113 von J. F. M. de Bongars an L. Freiherrn von Hohenhausen, Unterpräfekt in Eschwege, Werradepartement, 16.1.1812; vgl. ferner GERSMANN, Le monde des colporteurs parisiens.

71 Vgl. GStA PK, V. HA, Nr.698: Schreiben Nr. 113 von J. F. M. de Bongars an L. Freiherrn von Hohenhausen, 16. 1. 1812; GStA PK, V. HA, Nr. 699: Schreiben Nr. 1419 von J. F. M. de Bongars an den Grafen N. A. M. Rousseau de SaintAignan, französisch-kaiserlicher Minister an den sächsischen Hof in Weimar, 12.6. 1812 .

72 Vgl. RNB St. Petersburg, F 993 Arch. Westf., K. 14, Nr. 8041-8158, hier Nr. 8098: Schreiben Nr. 91 von Haas an J. F. M. de Bongars, 28. 2. 1813. 
Auch bei dem nachfolgend beschriebenen Fall ist nicht mehr eindeutig festzustellen, ob es sich um eine getarnte Karikatur handelte. Eine Medaille wurde zum Vehikel für einen antinapoleonischen Streich außergewöhnlicher Art. Cerfy, der Polizeiagent, der sich besonders in den Kasseler Gasthäusern umhörte und umsah, wusste am 15. Juli $1813 \mathrm{zu}$ berichten:

Gestern war die Rede im Bierhaus beym Pfeiler in der Wilde Mannsgaße von Münz fortes, da sagte, der Musikmeister von leichten Infanterie, daß er 3 Münzforten haben, darauf zog er seine Pörse heraus, nahm eine Metaille heraus, wo auf einer Seite gepregt ist, der Peüische Adler, und der französischer Adler, die beide sehen sich an, und oben siehet der deppelder Rußischen auf beide Adler herunter - Auf der andre Seite, stehet, denkmahl, der Schlacht bey Lützen - Der Musickmeister explecirte, dieses Sinnbild, wie folgete die beiden Adler sehen sich an, und wißen nicht was Sie thun wollen aber der Russischer Adler ist über beide -

Ich sagte zum Musickmeister, ob man diesen Metaille nicht hier haben kann, er sagte nein, sie sind zu Halle zu haben, ich fragte bey wem, dann ich wollte mir auch einen kommen laßen, darauf sagte er, und noch ein Chasseur Carabinier welcher dabey war, Es ist verbodene Ware, es sind mehrere unterm Batallion,

Es ist zu beobachten, als der obgedachtee, die Metaille mir zeigte, war sie an eine Couleurten Band gehäftet, wo noch etwas daran befestigt war, dieses aber lies er nicht sehen, sondern hielt es fest in der Hand, das ist sehr suspect.

Observation: Wann man obgedachtes Maitre de Musique durch seinen Officirs, citiren läßt, so wird er die Metaille auf seite thun - Durch ihm kann man den Verferdiger und die andern Inhaber erfahren ${ }^{73}$.

Es sind zwar keine Verhörprotokolle überliefert, die davon zeugen, ob der Musikmeister der leichten Infanterie und seine Kompagnons nun selbst verrieten, wie man in Halle an die Medaillen herankäme oder ob die dortige Lokalpolizei von selbst den Ursprung und die Verbreitung der Medaillen aufgrund der unvorsichtigen Wirtshausangaben der Kasseler Soldaten ausmachen konnte. Die darauffolgenden polizeilichen Maßnahmen lassen die Vermutung zu, dass sie wahrscheinlich ungewollt die Polizei auf die richtige Spur setzten:

des grenadiers de la garde et des chasseurs carabiniers acheterent la veille de leur départ de Halle des medailles en etain representant d'un coté les aigles de France, de Russie et de Prusse entourés des mots: Frankreich, Russland, Prussen, de l'autre est l'inscription suivante: Denkmal der grossen Schlacht bey Lützen den 1eu 2ten May 1813. Les militaire qui ont acheté de ces pieces, ont declaré qu'elles leur ont été vendues par une femme d'environ cinquante ans, de très petite taille, et fort maigre de figure, qui avoit une boutique mobile sur la place du marché et vendoit aussi des gravures representant les portraits de differents Souverains et autres personnages de marque ainsi que des chansons. [...]

Je vous charge Me. le sous Préfet de faire rechercher cette femme et de la faire interroger sur la source de la quelle elle a tiré ces medailes: Vous profiterez de cette occasion pour faire examiner tous les objets de sa boutique. Dans le cas ou l'on trouveroit parmis ses marchandises des objets qui la rendissent suspecte, ou qu'elle ne peut pas justifier de qui elle tient les medailles en question, vous la ferez mettre en

73 RNB St. Petersburg, F 993 Arch. Westf., K. 19, Nr. 11 919-12 074, hier Nr. 12014 : Bericht von C[erfy] an J. F. M. de Bongars, 15.7.1813. 
prison jusqu'à nouvel ordre de ma part. Dans tous les cas il faudra saisir toutes les medailles que l'on trouvera dans sa boutique, elle doit en avoir de plusieurs especes et il va sans dire que celle dont l'emprunte se conforme à nos principes politiques ne seront pas saisies ${ }^{74}$.

Hier ist ebenfalls eine Lücke in der Quellenüberlieferung zu dem Vorfall $\mathrm{zu}$ verzeichnen; allein aus einem Register über die Personen, die im Kastell in Kassel, dem Staatsgefängnis des Königreichs Westphalen, inhaftiert wurden, lässt sich rekonstruieren, dass die Verkäuferin der Medaillen auch deren Anfertiger angeben konnte. Johann Ernst Fischer, Graveur aus Halle, wurde am 29. Juli 1813 ins Kastell verbracht mit folgendem Eintrag zu seinem Vergehen: »Ayant gravé des medailles équivoques sur la bataille de Lutzen " ${ }^{75}$. Es ist anzunehmen, dass Fischer erst nach Auflösung des Königreichs Westphalen auf freien Fuß gesetzt wurde. Diese Art Taschen-Denkmal (monument de poche), die aus seiner Werkstatt stammte, hatte einen äußerst praktischen Charakter: Es ließ sich in der Hosentasche herumtragen und die Medaille erkannte man durch ihr Format nicht auf den ersten Blick als subversive Gedenkmedaille. Sie konnte auch als Münze unter anderen untertauchen, und obgleich sie im Format handhabbarer war als ein wirkliches Denkmal, besaß sie durchaus dessen Monumentalität ${ }^{76}$. Auch war es einfach, sie in der Runde unauffällig herumzureichen. Die Raffinesse im Fall der Gedenkmedaille oder des Taschen-Denkmals bestand auch darin, dass sie sich der offiziellen Embleme bediente, damit eng entlang den verherrlichenden Darstellungen napoleonischer Triumphe argumentierte und dennoch diese staatseigenen rhetorischen Mittel letztlich zu einem Spottbild umfunktionierte ${ }^{77}$.

74 RNB St. Petersburg, F 993 Arch. Westf., Nr. [13 851,1], Registre de Correspondance du Sécrétariat général de la haute police, 1.7.-24.9.1813: Schreiben Nr. 1846 an den Unterpräfekten in Halle, 21.7. 1813.

75 RNB St. Petersburg, F 993 Arch. Westf., Nr. [13 852], Registre des personnes arrêtées, où sont indiqués les $\mathrm{NN}$ d'ordres, date de l'entrée, les noms et prénoms des susdites personnes, les signalements, les motifs de l'arrestation, les décision, dates de la sortie et les observations différentes la-dessus, depuis le 1811, 1812, 1813: Eintrag Nr. 323. Um 1800 lieferte Fischer in Zusammenarbeit mit dem bereits in Kapitel B II. erwähnten Buchdrucker Dreyssig ein einmaliges pädagogisches Werk, ein »Realienbuch für die Jugend «, das Buch und Zinnfiguren kombinierte. Vgl. SCHRAUDOLPH, Eisvogel trifft Klapperschlange, bes. S. 25, 44.

76 Vgl. zur Denkmalkultur mit medialem und monumentalem Charakter im 19. Jh., die auf die napoleonische Ära zurückgeht: KelleR, SCHMID (Hg.), Vom Kult zur Kulisse; vgl. auch NIPPERDEY, Nationalidee und Nationaldenkmal; BISCHOFF, Denkmäler der Befreiungskriege; KosELLECK, Kriegerdenkmale, S. 255-276; LURZ, Denkmäler der Befreiungskriege; DERS., Kriegerdenkmäler; KOSELLECK, JeISMAnN (Hg.), Der politische Totenkult; KoRfF, Materialität der Erinnerung; Berding, Heller (Hg.), Krieg und Erinnerung; Gersmann, Wolfrum (Hg.), Totenkult und Erinnerungskultur.

77 Das Format der Münzen wurde auch von den kaiserlichen Sinnproduzenten in der napoleonischen Propaganda ausgiebig verwendet. Vgl. OWZAR, Zwi- 


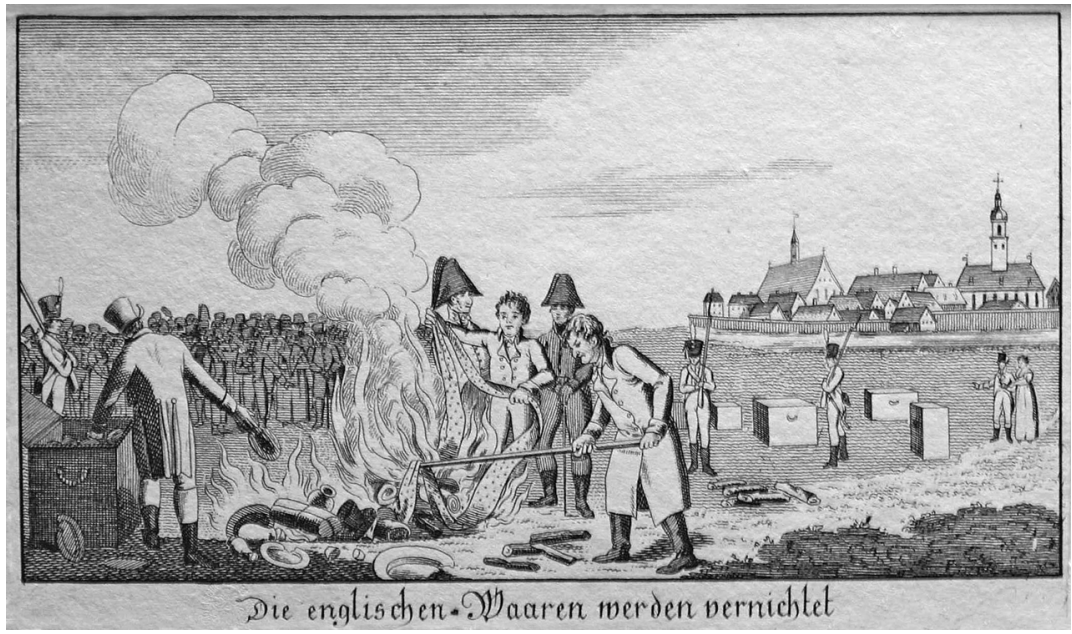

Abb. 20: C. G. H. Geißler, Die englischen Waaren werden vernichtet, Leipzig, 1810, Radierung, $11 \times 19 \mathrm{~cm}$, SML, Nap. II 112 .

Wegen der Kontinentalsperre fanden öffentliche Verbrennungen von englischen Waren allerorten im Königreich statt ${ }^{78}$. Ein westphälischer Zeitgenosse berichtet: »Wir haben zu Halberstadt einen Berg solcher Fabrikate auf offenem Markte in Flammen aufgehen sehen; es war ein politisches autodafé(Glaubensschauspiel) und zugleich ein warnendes Exempel des Kontinentalsystems, d.i. der Absperrung des ganzen europäischen Festlandes gegen den Verkehr mit England «"79.

In einem weiteren Sinn, wenn auch nicht satirisch unterlegt wie bei der Gedenkmedaille, bediente sich auch die oben erwähnte westphälische Karikatur mit der Magd, die französisches und westphälisches Schriftgut ins Feuer gibt, aus dem »Handlungsrepertoire« der westphälischen Herrschaft ${ }^{80}$.

Armin Owzar stellt in Bezug auf den sinkenden Stern Napoleons in Europa fest, dass »sämtliche Topoi und Strategien, deren sich die napoleonischen

schen Gottesgnadentum und Verfassungspatriotismus, S. 136; ZEITZ, Napoleons Medaillen. Andere Spott- und Volksmedaillen enthielt die Sammlung Schwering, vgl. ZEITZ, Die schönen Kehrseiten S. 388, 390.

78 Vgl. König Lustik!?, S. 450 f., Kat. 382; BUTTKEREIT, Zensur und Öffentlichkeit, S. 33 .

79 NAGEL, Kriegsbilder aus der Heimath, S. 80; vgl. Bulletin des Lois, Erster Band, ${ }^{2}$ 1810, Nr. 16 - Dekret vom 5. Februar 1808, S. 412-414. Decret, welches verordnet, daß die zu Marburg gefundenen engl. Waaren verbrannt werden sollen; Le Moniteur westphalien, Nr.20, 11. Februar 1808, S. 79; ibid., Nr. 169, 29. November 1810, S.744f.; ibid., Nr. 191, 24. Dezember 1810, S. 839; ibid., Nr. 197, 31. Dezember 1810, S. 862 f.; SCHELLER, Jeromiade, S. 16f.; MÜLLER, Kassel seit siebzig Jahren, Bd. 1, S. 33 f.; THIMME, Die inneren Zustände, Bd. 2, S. 189; [LEHSTEN-DingELSTÄDT], Am Hofe König Jérômes, S. 54.

80 ALGAZI, Kulturkult. 
Sinnproduzenten bedient hatten, [...] nun von den Kritikern aufgegriffen und gegen Napoleon eingesetzt [wurden], vorzugsweise auf Karikaturen $\aleph^{81}$.

Um auf die Tabaksdosen, Pfeifen und Gedenkmedaille zurückzukommen: Wenn es möglich war, auf einer Medaille seinen Spaß über das Zeitgeschehen und die Politik zu treiben, so waren ganz andere Formate für Satiren und kritische Darstellungen ebenfalls denkbar, die das breite Entwicklungsspektrum weg von der klassischen Karikatur aufzeigen.

\section{Weitere staatskritische und satirische Darstellungen und ihre Gemeinsamkeiten mit den Karikaturen}

Folgende antinapoleonische Angelegenheit meldete Bongars im November 1812 dem Generalpolizeikommissar Guntz zu Braunschweig:

L'on m'assure $[\ldots]$ qu'il existe à Brunswick un cabinet ou l'on expose à la vue du public les figures et portraits des bourbons emigrés et de Guillaume Tell, l'assassinat d'un Général françois par le peuple de Rome des scenes representant des victoires remportées par les insurgés espagnols sur les françois, et il se fabrique dans la manufacture de cuir boulli dont la succursale est à Berlin, des caricatures dans le plus mauvais esprit, vous vous assurerez si ce cabinet existe dans Brunswick alors vous le ferez fermer, et si les portraits et tableaux c'y dessus designés y existent reéllement vous les confisquerez et vous signifirez au proprietaire de ces objets que si il se permet encore d'exposer de pareils objets qu'il sera puni de la maniere la plus sévère ${ }^{82}$.

Diese Informationen über eine Art Wachsfigurenkabinett in Braunschweig bezog Bongars direkt aus Frankreich, wo die französische geheime Polizei Agentenberichte darüber vorliegen hatte ${ }^{83}$.

Man sieht: Von der Gedenkmedaille bis hin zu den tableaux des Wachsfigurenkabinetts existierten vielerlei Formen, die napoleonische Herrschaft und das politische Zeitgeschehen satirisch zu betrachten, die den engen papiernen Rahmen einer Karikatur sprengten, aber teilweise auf einer ähnlichen Inszenierung beruhten oder eine verwandte List bemühten ${ }^{84}$. Als wichtiger Bestandteil oder entscheidende Beilage zu einer Karikatur gehörten oftmals ein Spruch, ein paar Verse, eine Erzählung, die das Potential der Karikatur zu katalysieren wussten und ihr zum Ausbruch in die Welt ver-

81 OwZAR, Der alte Schein des neuen Reiches, S. 160; vgl. Duprat, Symboles et allégories, S. 142. In manchen Fällen griffen die Karikaturen sogar dem ikonographischen Register Napoleons vor. Vgl. ibid., S. 145.

82 RNB St. Petersburg, F 993 Arch. Westf., Nr. 13 848, Registre de correspondance du bureau de la police secrette, 5. 11.-30.1.[1812]: Schreiben Nr. 2087 von J. F. M. de Bongars an F. T. de Guntz, 26. 11.1812.

83 Vgl. ibid., Schreiben Nr. 2091 von J.F. M. de Bongars an den Grafen P.A. von Fürstenstein, Ministre Secrétaire d'État et des Relations extérieures, 26. 11. 1812. Weiterführend vgl. Gersmann, Revolution im Musée Grévin; KnaUer, »Tableaux patriotiques«.

84 Vgl. weiterführend über die Inszenierungen in den tableaux des Pariser Wachsfigurenmuseums, Musée Grévin, HermanNs, Musée Grévin. 
halfen. Auch ermöglichten diese schriftlichen Ergänzungen ihre Aneignung durch ein breites Spektrum der Gesellschaft. Nicht anders stand es mit den Porträts, die der Maler Körber zu Bernburg angefertigt hatte. Der Polizeiagent Garagnon stellte in seinem Bericht vom 6. 1. 1813 fest:

J'ai aussi entendu que la Police de Magdebourg s'est fait délivré les peinture que le Sieur Körber à Bernbourg a fait du tems que des gens du commun peuple passoit par cette ville pour servir de gardes dans les hopitaux de la grande armée. Ils les nommoient par dérision: Employé du grand Quartier Général.

Ce Koerber est digne d'une forte leçon, car c'est un de ces babillards politiques. On ajoute qu'il doit avoir dit dans son interrogatoire par rapport à ces peintures, qu'un peintre avoit la permission de copier tout ce qui lui paroissoit nouveau. Mais pourquoi le fameux Breymann a-t-il voulu avoir copie de ces portraits, si c'étoit quelque chose de peu d'importance? On doit avoir même trouvé sous quelques uns des portraits: Notre garde de l'Empereur, \& il doit avoir dit pour sa défense, que c'étoit un de ses élèves qui avoit écrit ces mots ${ }^{85}$.

Die Gemälde mit den Porträts von Anwärtern auf die Wächterstellen der Feldlazarette der Grande Armée hätten an sich keine Satire darstellen können, hätte sie nicht ihr Autor »Employés du grand Quartier Général « betitelt und im Einzelnen auch so beschriftet. Wie oft bei den Karikaturen war der erläuternde Textzusatz in diesem Fall ganz entscheidend für die satirische Verwandlung der Porträts ${ }^{86}$.

\section{Erzählte Karikaturen und die innere kollektive Bild(referenz)welt der Westphalen}

Karikaturen, Druckbilder und andere satirische Darstellungen kamen oftmals erst durch das zum Vorschein, was man sich über sie erzählte. Entscheidend war, dass man sie überhaupt weitererzählte: Damit spielten die westphälischen Staatsbürger eine maßgebliche Rolle in der Entfaltung der Wirkung der Karikaturen und anderer zeitgenössischer Satiren. Für das Medium »Karikatur« stellte zum einen die Interdependenz von Bild und Schrift einen zentralen Faktor dar, zum anderen ermöglichte der Transfer von der Ikonographie zur Mündlichkeit ihre Verbreitung in breiten Schichten der Gesellschaft.

Wendet man sich den Darstellungen in den Karikaturen selbst zu, so fasziniert sehr bald, wie schon eingangs dargestellt, die Vielschichtigkeit im Bezug auf die darin entfalteten rhetorischen Mittel, Sprachbilder, stilisierte Mündlichkeit und Schriftlichkeit - Untertitel, Sprechblasen, beschrifte-

85 RNB St. Petersburg, F 993 Arch. Westf., K. 16, Nr. 9851: Rapport von Garagnon, Polizeiagent, an J. F. M. de Bongars, 6. 1. 1813. Körbers Gemälde könnte in einem Interpretationszusammenhang mit Geißlers Bild »Trümmer der französischen Armée« gebracht werden, siehe Abbildung 12, S. $216 f$.

86 L'anti-Napoléon, S. 6. 
te Seifenblasen, Aufschrift um den Hals gehängt ${ }^{87}$. Die Sprache hatte eine Schüsselrolle innerhalb des bildlichen Mediums zu spielen. Auf der Ebene ihrer Verbreitung zeigt sich das Medium der Karikatur ebenfalls stark verankert in einem komplexen und vielschichtigen kommunikativen Gefüge der Westphalen aus Mündlichkeit und Schriftlichkeit. Als Bild allein wären sie nicht so weit verbreitet worden: Das Reden und Erzählen darüber machte die Karikaturen erst richtig bekannt unter den Westphalen. Die von den Karikaturen oder ihren Nacherzählungen verbreiteten Sprachbilder scheinen das mentale politische Bewusstsein der Westphalen in breitem Umfang untermalt zu haben. Den Polizeibeamten wurden zumindest die Karikaturen in ihren erzählten Versionen noch mehr zum Problem als ihre papierenen Originale, denn der mündliche Austausch der westphälischen Staatsbürger war wesentlich schwieriger aufzuhalten, als man ein Druckbild dingfest machen konnte.

Im Fall der Karikaturen hat man es mit einem Medium zu tun, das seinen Ursprung eindeutig bei den versierten und gebildeten Napoleonkritikern hatte, wobei es allerdings eine derartige Popularität erlangte, dass es durchaus ein weites Spektrum der Gesellschaft erreichte. Durch die Nacherzählungen der Karikaturen erfolgte sogar eine eigenwillige Aneignung des Mediums durch weniger gebildete und politisch engagierte Teile der Bevölkerung - Zusätze und kreative Umwandlungen waren nicht ausgeschlossen. Hier fand ein erfolgreicher Transfer von den Eliten hin zur Basis der Gesellschaft und eine Übernahme in die Volkskultur statt ${ }^{88}$. Mit der Verwendung

87 Über die verschiedenen Modi der schriftlichen Angaben in den Karikaturen vgl. L'anti-Napoléon, S. 8. Tschopp macht für die Flugblätter und Flugschriften des 17. Jh. eine Grundmotorik aus, die sich in den Karikaturen des anfänglichen 19. Jh. offensichtlich wiederholte: »verbale und graphische Darstellung [gelangen] zur Deckung, [...] bildliche Rede und sprechendes Bild [verbinden sich]. Neben der Bild-Text-Beziehung als Relation zwischen einer wörtlichen Äußerung und einem visuellen gestalteten ikonographischen Ensemble kommt so in den vorgängig untersuchten publizistischen Manifestationen auch eine sinnere Bild-Text-Beziehung zum Tragen, die sich sprachlicher Einbildungskraft verdankt «. TsCHOPP, Rhetorik des Bildes, S. 95.

88 Über die Karikaturen als Mittel zur Popularisierung ihrer antifranzösischen Einstellung in der Hand von Vertretern eines frühen deutschen Nationalismus vgl. u.a. LÜSEBRINK, REICHARDT, "Kauft schöne Bilder, Kupferstiche...«, S. 21, 33. Im Königreich Westphalen fällt auf, dass mehr antinapoleonische als antiwestphälische Karikaturen im Umlauf waren. Dies weist auf den europäischen Produktionsrahmen hin und auf die Produktion, die auf eine Elite zurückgeht. Die Bildnachrichten werden von Lüsebrink und Reichardt neben den mündlich zirkulierenden Nachrichten, Erzählungen und Liedern als das herausragende Informationsmedium erkannt, für jene Bevölkerungsschichten, die des Lesens und Schreibens unkundig waren. Vgl. ibid., S. 14. Vgl. dort auch über die Bänkelsänger, die auf ihren Reisen mit Bildtafeln und Liedern von politischen und gesellschaftlichen Ereignissen berichteten. Ein westphälischer Bänkelsänger war der von Pröhle erwähnte »invalide Rauchheld aus Neuhaldensleben«, der »noch 
unter anderem von karnevalistischen Themen und dem Einsatz von Redensarten und von volksnahen Sprachbildern, die in Verbindung mit der Alltagskultur des »kleinen Mannes« standen, suchten die Karikaturisten bewusst Anschluss an die Volkskultur. Napoleon wurde beispielsweise als Bäcker oder als Fleischer dargestellt und über diese einfachen Tätigkeiten zurückgeholt in die Lebens- und Arbeitssphäre der gesellschaftlichen Schichten, die sich ihr Brot mühsam verdienen mussten. Die Karikaturisten leisteten einen wenn nicht Übersetzungs-, so doch Umwandlungsprozess ihrer fundierten Staatskritik zu einer "propagande en images ${ }^{89}$. Die überlieferten Beispiele aus der Geschichte des Königreichs Westphalen zeigen in vielerlei Hinsicht, wie dies auch hervorragend gelang und lassen die Aussage $\mathrm{zu}$, dass diese satirischen antinapoleonischen Darstellungen allesamt zur Politisierung der Westphalen und zur Schärfung ihres politischen Bewusstseins beigetragen haben ${ }^{90}$.

vor zehn Jahren seine bunten Erlebnisse auf Märkten und Straße des ehemaligen Königreichs Westphalen von der Saale bis zur Bode" absang. PRÖHLE, Die Fremdherrschaft, S. 15, vgl. dort mehr zu diesem Rauchheld S. $15 \mathrm{f}$.

89 L'anti-Napoléon, S. 7, vgl. S.6, 10, 24, 27; vgl. FrEIST, Wirtshäuser, S. 212; TsCHOPP, Rhetorik des Bildes, S. 96.

90 Vgl. LÜSEBRINK, REICHARDT, »Kauft schöne Bilder, Kupferstiche...«, S. 26. 